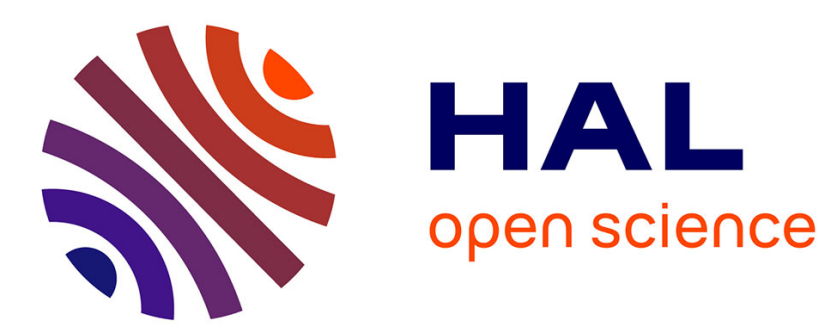

\title{
Impact of Simulation Environment in Performance Evaluation of Protocols for WSNs
}

Therèse Affoua Aby, Marie-Françoise Servajean, Nadir Hakem, Michel Misson

\section{To cite this version:}

Therèse Affoua Aby, Marie-Françoise Servajean, Nadir Hakem, Michel Misson. Impact of Simulation Environment in Performance Evaluation of Protocols for WSNs. 16th International Conference on Ad Hoc Networks and Wireless, ADHOC-NOW 2017, Sep 2017, Messina, Italy. hal-01630958

\section{HAL Id: hal-01630958 https://hal.science/hal-01630958}

Submitted on 18 Sep 2018

HAL is a multi-disciplinary open access archive for the deposit and dissemination of scientific research documents, whether they are published or not. The documents may come from teaching and research institutions in France or abroad, or from public or private research centers.
L'archive ouverte pluridisciplinaire $\mathbf{H A L}$, est destinée au dépôt et à la diffusion de documents scientifiques de niveau recherche, publiés ou non, émanant des établissements d'enseignement et de recherche français ou étrangers, des laboratoires publics ou privés. 


\title{
IMPACT OF SIMULATION ENVIRONMENT IN PERFORMANCE EVALUATION OF PROTOCOLS FOR WSNS.
}

\author{
Affoua Therese Aby ${ }^{1}$, Marie-Françoise Servajean ${ }^{1}$, \\ Nadir Hakem ${ }^{2}$, and Michel Misson ${ }^{1}$ \\ ${ }^{1}$ UCA (Université Clermont-Auvergne), LIMOS, CNRS, UMR 6158, \\ BP 10448, F-63175 Aubière, France, \\ ${ }^{2}$ UQAT (Université du Québec en Abitibi-Temiscamingue), \\ LRTCS, Val d'Or, Qc, Canada. \\ abyeesiee-amiens.fr,m-francoise.servajean@uca.fr \\ nadir.hakemeuqat.ca, michel.missoneuca.fr
}

\begin{abstract}
Wireless sensor networks are increasingly used in many emerging applications. This type of network is composed of hundreds of low-cost sensor nodes, but with a limited budget batteries, low communication range, limited throughput, reduced computing power, low memory and low storage capacity. Communication protocols are proposed in the literature to deal with technical challenges coming from low intrinsic resources of sensor nodes. In most of these studies, simulations comparing a proposed protocol with other existing protocols are performed to show that the proposed protocol provides overall better performance. However, the environmental specification that made these comparisons is very often neglected or non-existent. In this study we show that it is essential to have the simulation environment very well defined before considering whether a protocol provides better performance than others. To do this we use two duty cycle MAC protocols, the standard IEEE 802.15.4 and SlackMAC (a protocol that we proposed). The aim of the paper is not to make an exhaustive comparison of protocols. We intuitively know that SlackMAC provides a better overall performance than the standard. What we are trying to show is the gap between performances according to simulation conditions. We will mainly focus on the topologies used and the capture effect. The results draw attention to the fact that it is essential to clearly define the simulation environment and also to reconcile the chosen conditions with the results when comparing the performances of two protocols.
\end{abstract}

Keywords: WSNs; QoS; performance evaluation; simulation conditions; topologies impact; capture effect impact

\section{Introduction}

Wireless Sensor Networks (WSNs) have attracted great interest in the last decade. This explains the rich and active research about communication protocols for WSNs in order to deal with technical constraints related to sensor nodes. In most of these studies, in 
the absence of real conditions to validate the proposed protocols, many simulations are carried out to evaluate their performances. Mostly, these simulations are done with performance comparisons of proposed protocols compared to existing one to show that the new protocol provides better performance. However, sometimes the level of detail on the results surpasses the description of the simulation conditions used to obtain them.

In this paper we raise the curtain on this issue and we draw attention regarding the quantitative results of simulation when communication protocols are compared. Let us take the case of works that conduct to establish efficient mechanisms to ensure a long lifetime to the network. We take as examples energy-efficient MAC protocols based on sequences of active periods (during which the radio module is on) and sleep periods (during which it is off) called duty cycle. The duty cycle represents the proportion of the active period over the total duration of the cycle (active period + sleep period). The main energy-saving MAC protocol based on this mechanism is the standard IEEE 802.15.4 [1] in beacon-enabled mode. In this type of protocols nodes agree on a common calendar for their periods of activity and sleep. This category is called synchronous duty-cycle MAC protocols. Other categories based on an asynchronous mechanism are also proposed in the literature. In these second categories, nodes do not have a common calendar for their period of activity and sleep. Most of these protocols are proposed as improvements to some existing protocols. However, there is a lack of important information on simulation conditions such as: radio frequency, antenna, propagation model, transmission power, topology information, data traffic information, capture effect, etc.

These omission do not allow to judge the relevance of the results and also does not allow reproducibility. There are studies that show the impact of different propagation models and topologies on simulation results as in [2] and [3]. However, to the best of our knowledge, no studies have shown these impacts with such a high level of detail as that in this paper. We use two energy-efficient MAC protocols, the standard IEEE 802.15.4 [1] and SlackMAC [4,5] (a protocol that we have proposed). The aim of the paper is not to make an exhaustive comparison of protocols. We know intuitively that SlackMAC generally provides better performance than the standard. What we are trying to demonstrate through intensive simulations is the diversity in the performance gaps according to simulation conditions. Mainly, we will focus on the topologies used and the capture effect.

This paper is organized as follows. In section 2, we make a summary of duty cycle MAC protocols and take note of the levels of detail on the missing simulation conditions. In section 3, we analytically compare the two protocols that serve tests in our study and give the technical details of our study. In section 4, we show simulation results from this comparative study. Finally, we conclude our work in section 5.

\section{State-of-the-art}

The MAC protocols of the literature based on the duty cycle mechanism can be classified into two main categories such as synchronous duty cycle MAC protocols and asynchronous duty cycle MAC protocols. 


\subsection{Synchronous duty cycle MAC protocol}

In synchronous duty cycle MAC protocols synchronization can be global or local.

In the case of global synchronization, all nodes share a common schedule for their periods of activity and inactivity. The main MAC protocol based on this mechanism is the standard IEEE 802.15.4 [1] in beacon enabled mode. This is also the case of protocol in [6] and LO-MAC (Low Overhead MAC) [7].

In the case of local synchronization, the nodes are synchronized by neighborhood, very often according to a tree topology as a function of their position relative to the sink. One of the first protocols based on this mechanism is S-MAC (sensor-MAC) [8] then D-MAC (Data-gathering MAC) [9] and TreeMAC [10]. Improvements of these protocols are proposed in ID-MAC [11], DW-MAC (Demand Wakeup MAC) [12], DSF (Dynamic Switch-based Forwarding) [13] and iCore [14].

\subsection{Asynchronous duty cycle MAC protocol}

In asynchronous duty cycle MAC protocols, nodes do not have a common calendar for their activity and sleep periods. A distinction can be made between sender-initiated and receiver-initiated MAC protocols.

In sender-initiated MAC protocols, most of the communication load is supported by sender nodes. The main protocol based on this principle is B-MAC protocol Berkeley $M A C)$ [15]. In B-MAC, a sender sends a long preamble before sending data frame. XMAC [16] is one of the first improvement of B-MAC. Many other protocols such as BoX-MAC [17] and OSX-MAC [18] have been proposed thereafter.

Unlike sender-initiated MAC protocols, in receiver-initiated MAC protocols, most of the communication load is supported by the receiver nodes. In receiver-initiated MAC protocols the receiver initiates the communication by sending a beacon frame to express its ability to receive data frame. RI-MAC [19] is the main protocol based on this mechanism. RIX-MAC (Receiver-Initiated X-MAC) [20], ERI-MAC [21], OCMAC [22] and protocol in [23] are improvement of RI-MAC.

Other hybrid MAC protocols that are both sender-initiated and receiver-initiated have been proposed to balance the communication load on the sender and receiver nodes. This is the case of protocol in [24] and SlackMAC [4,5].

\subsection{Overview of simulation conditions}

In most of the protocols cited above comparisons by simulation on NS2 [25] are performed to show that the proposed protocols provide better overall performance than those of the existing. Table 1 gives a summary of simulation conditions description used in these comparisons. These simulation conditions essentially concern radio frequency, antenna (type, gain, height), propagation model, transmission power (noted TX power in table 1). It is also important to provide information about topology used (type, size, transmission range that provides connectivity, number of nodes, and sink position). Parameters such as receive power threshold and carrier sense threshold (noted RXThresh_ and CSThresh_), and capture threshold (noted CPThresh_) are all equally important. 
Traffic information such as type, size in byte and generation period of data packets are also required.

It can see from Table 1 that these important elements of simulation conditions are often not fully defined and sometimes non-existent.

Table 1: Overview on specification of NS2 simulation conditions

\begin{tabular}{|c|c|c|c|c|c|c|c|c|c|c|c|c|}
\hline $\begin{array}{c}\text { Simulation conditions / } \\
\text { Protocols }\end{array}$ & 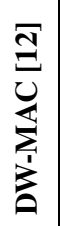 & D & 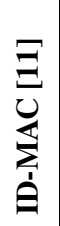 & 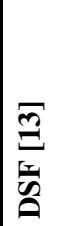 & 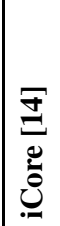 & 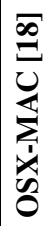 & 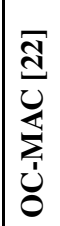 & 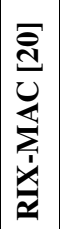 & $\overline{\tilde{d}}$ & 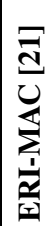 & $\underset{\mathrm{J}}{\Xi}$ & 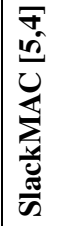 \\
\hline Radio Frequency & $\sqrt{ }$ & $\sqrt{ }$ & X & $\sqrt{ }$ & X & X & X & X & X & $\sqrt{ }$ & X & X \\
\hline Antenna: -type -gain -height & $1 / 3$ & $1 / 3$ & X & $\sqrt{ }$ & X & X & X & $1 / 3$ & X & X & X & X \\
\hline Propagation model & $\sqrt{ }$ & $\sqrt{ }$ & X & X & $\sqrt{ }$ & X & X & $\sqrt{ }$ & $\sqrt{ }$ & X & $\sqrt{ }$ & $\sqrt{ }$ \\
\hline TX power & $\sqrt{ }$ & $\sqrt{ }$ & $\sqrt{ }$ & X & X & $\sqrt{ }$ & X & $\sqrt{ }$ & X & $\sqrt{ }$ & $\sqrt{ }$ & $\sqrt{ }$ \\
\hline $\begin{array}{l}\text { Topology: -type -size -TX range } \\
\text {-number of nodes -sink position }\end{array}$ & $\sqrt{ }$ & $\sqrt{ }$ & $\sqrt{ }$ & $3 / 5$ & $\sqrt{ }$ & $1 / 5$ & $\sqrt{ }$ & $3 / 5$ & $\sqrt{ }$ & $\sqrt{ }$ & $\sqrt{ }$ & $\sqrt{ }$ \\
\hline -RXThresh_-CSThresh_ & X & $\sqrt{ }$ & X & X & X & $\sqrt{ }$ & X & $\sqrt{ }$ & X & X & X & X \\
\hline Traffic: -type -size -period & $\sqrt{ }$ & $2 / 3$ & $2 / 3$ & $1 / 3$ & $1 / 3$ & $1 / 3$ & $2 / 3$ & $\sqrt{ }$ & $2 / 3$ & $2 / 3$ & $2 / 3$ & $2 / 3$ \\
\hline Capture Threshold & X & X & $\mathrm{X}$ & X & X & X & X & X & $\mathrm{X}$ & $\mathrm{X}$ & $\mathrm{X}$ & $\mathrm{X}$ \\
\hline
\end{tabular}

\section{Study framework}

In this section we first describe the operating mechanism of the reference synchronous MAC protocol described in the standard IEEE 802.15.4 [1] in beacon enabled mode and the asynchronous MAC protocol SlackMAC [5,4]. Then we give the technical details of the implementation of our comparative study.

\subsection{Description of the operating mechanism of IEEE 802.15.4 and SlackMAC}

In the beacon enabled mode of the standard IEEE 802.15.4, the medium is accessed using the slotted CSMA/CA (Carrier-Sense Multiple Access with Collision Avoidance) algorithm. All nodes wake up periodically together and share a common activity throughout the $S D$ (Superframe Duration) period and change to sleep mode the rest of the $B I$ (Beacon Interval) period.

The left side of Figure 1 shows an example of the activity and sleep mechanism in the standard IEEE 802.15.4 with a duty cycle of $25 \%\left(\frac{S D}{B I} \times 100\right)$ for three nodes $\left(n_{1}\right.$, 

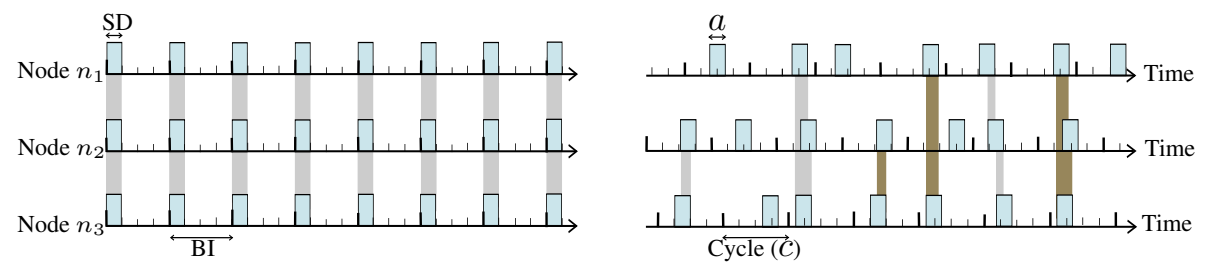

Fig. 1: Example of activity of three nodes $n_{1}, n_{2}$ and $n_{3}$ in range, with a duty cycle of $25 \%$ for respectively the standard IEEE 802.15.4 (left) and SlackMAC (right).

$n_{2}$ and $\left.n_{3}\right)$ in range. It can be seen that all the activity of the nodes is concentrated on the period $S D$. This reduces the communication time given to each node and increases the risk of collision depending on the number of nodes in competition to access the medium.

In SlackMAC, the medium is also accessed using the slotted CSMA/CA algorithm. The right side of Figure 1 shows an example of activity cycle for three nodes $\left(n_{1}, n_{2}\right.$ and $\left.n_{3}\right)$ in range with a duty cycle of $25 \%\left(\frac{a}{c} \times 100\right)$ in SlackMAC. Unlike the standard in SlackMAC, initially all nodes choose their activation times uniformly at random in the cycle. When a node chooses a time that yields to successful communications (reception or transmission of a frame), it memorizes it and the probability to choose this time increases, as can be seen in darker towards the right of Figure 1. It is noted that the activity of the nodes can be distributed over the whole cycle unlike the standard. Indeed, on average there are few active nodes at the same time, which reduces the risk of collision and allows a higher communication time per nodes than the standard for the same duty cycle as shown in [4].

According to such conditions, it is intuitively known that SlackMAC will ensure overall better performance than the standard. However, what we are trying to show in what follows is the diversity in the performance gaps according to simulation conditions.

\subsection{Technical details}

In this part we detail the technical environment used for our simulations. We want to highlight the fact that in a deployment study and a priori evaluation of its performance by simulation of WSNs, it is essential to be attentive and precise on the description of simulation conditions. In a simulation process we know how the choice of the propagation model is important for the relevance of the results. In this paper we will consider that this choice has been made at best and we focus on the impact of the chosen type of topology and on the effects of the given value of capture threshold parameter, often neglected in literature. The topology (or distance between nodes), the propagation model, and the capture threshold are three elements concerned to distinguish a collision of a successful reception. In a first step we propose a small synthesis of the topologies usually exploited but not often justified and/or not sufficiently detailed. On the second hand we provide some clarification on why it is important to specify the capture effect. 
Topology production strategy In addition to specifying the propagation model and its associated parameters, it is also important to give the rules that allowed to generate the topology (or topologies) used for the simulation. Generally, in the used WSNs topologies for communication protocol comparison tests, nodes are positioned according to a grid or randomly distributed over a given area.
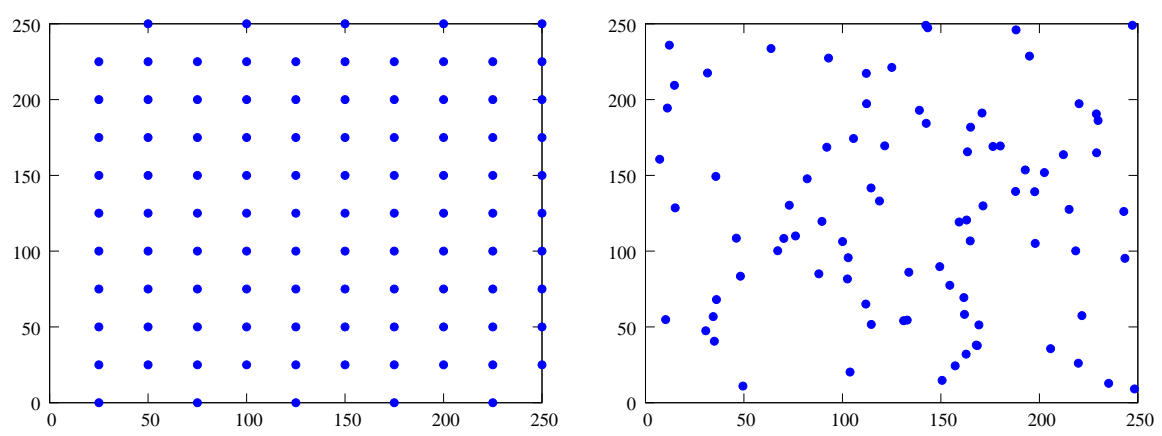

Fig. 2: Topology of 100 nodes on square area of $250 \mathrm{~m}$ x $250 \mathrm{~m}$ with respectively a grid positioning (left) and random positioning (right).

Figures 2 and 3 illustrate respectively the topologies in square and pseudo-linear, with in each case, one hundred nodes are positioned both in grid and randomly.

The random positioning is more closer to a realistic positioning. However, the concept of random position can change depending on how the placement is carried out. Indeed, to randomly place $\mathrm{N}$ nodes on a given area by hand, the person will try to cover the whole area and avoid nodes overlapping. For the same surface $S$ and for the same number of nodes, the topologies obtained by a generator of random positions $(x, y)$ lead to much greater diversity of solution but are not necessarily more representative for WSNs field. It can have less covered places than others, overlays and nodes that are too distant from others. To avoid the latter two situations, once the positions are
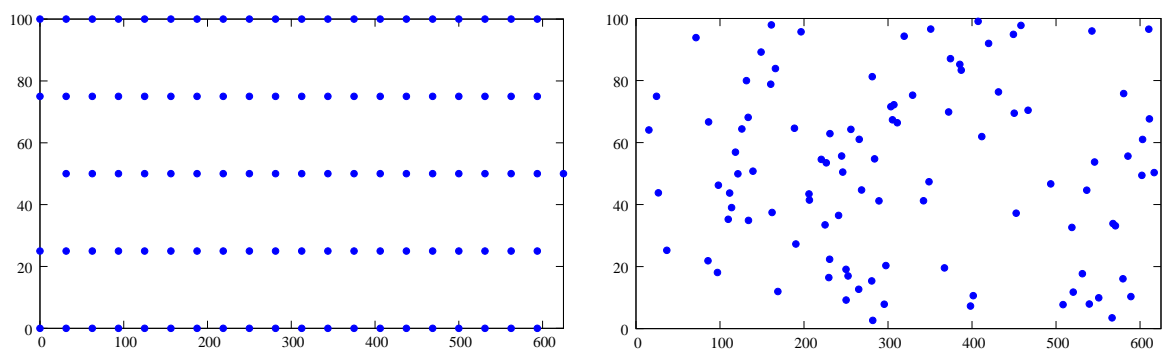

Fig. 3: Topology of 100 nodes on square area of $625 \mathrm{~m}$ x $100 \mathrm{~m}$ with respectively a grid positioning (left) and random positioning (right). 
randomly drawn, we use two filters in the control of network connectivity by Prim's algorithm (developed by Robert C. Prim (1957)). We set a minimum value of $1 \mathrm{~m}$ and a maximum of $50 \mathrm{~m}$ between any neighbors nodes. The surface of the coverage area $\left(62500 \mathrm{~m}^{2}\right)$, the propagation model, the data packets production, the total number of nodes and the percentage of source nodes are identical in each case. We consider two forms of topologies (square of size $250 \mathrm{~m}$ x $250 \mathrm{~m}$ and pseudo-linear of size $625 \mathrm{~m} \mathrm{x}$ $100 \mathrm{~m}$ ) and for each type, nodes are positioned both in grid and randomly. In any case, the sink is located at the top right corner of the area.

In this work, our field of investigation is thus reduced to simulations on these 4 types of topologies (see Figures 2 and 3) with different values of capture threshold.

Capture effect The capture effect relates to the simultaneous reception of several frames with power levels such that the reception of one of them is possible. When a frame $f$ is received with a power $P_{f}$ greater than or equal to the sum of the powers of the other $k$ frames received at the same time plus a capture threshold fixed, the frame $f$ is correctly decoded by the receiver. The capture threshold is the minimum power ratio in $\mathrm{dB}$ which enables the receiver in case of simultaneous reception to decode the strongest signal correctly. Therefore, if the difference between the power of a received signal and the sum of power of the other signals is greater than this threshold, reception is considered as a capture and the dominant signal is decoded correctly. Otherwise, the simultaneous reception will cause a collision and the signals can not be interpreted. This capture threshold has a direct impact on the collision rate of frames when the traffic subjected to the network becomes very significant.

\section{Results}

We carried out several simulations to show the diversity in the results as a function of simulation conditions for protocols SlackMAC [4,5] and the standard IEEE [1]. We first describe the simulation environment and then compare the two MAC protocols.

\subsection{Simulation parameters}

Our simulations are performed using the network simulator NS-2 [25]. Global simulation parameters are given in Table 2. We use 10 of each type of topology of 100 nodes described in section 3 (see figures 2 and 3 ). We generate a convergecast communication (from the nodes to the sink), for 30 source nodes located randomly in the network. These source nodes perform periodic measurements and route them via other nodes to the sink. Nodes have a duty-cycle of $1 \%$ and the global cycle is $5 \mathrm{~s}$ (that is, nodes are active during $A=50 \mathrm{~ms}$ every $C=5 \mathrm{~s}$ ). The same gradient-based routing protocol is used to route packets hop by hop towards the sink for both MAC protocols. All presented results, in each case are averaged over 10 repetitions per topology for the 10 topologies. We compute transmission power (in $\mathrm{dBm}$ ) using outage probability method (defined in $[26,27])$. For transmission range of $50 \mathrm{~m}$ and shadowing parameters defined in Table 2, the equivalent transmission power which can ensure stability of the radio links for $95 \%$ of the reception between two nodes is equal to $10.5342501084 \mathrm{dBm}$. This value is used as a transmission power in all simulations. 
Table 2: Global simulation parameters

\begin{tabular}{|l|c|}
\hline Topologies area & $250 \mathrm{~m}$ x 250 m and $625 \mathrm{~m} \mathrm{x} 100 \mathrm{~m}$ \\
\hline Transmission range & $50 \mathrm{~m}$ \\
\hline Number of nodes & 100 \\
\hline Number of source nodes & 30 \\
\hline Radio frequency & $2.4 \mathrm{GHz}$ \\
\hline Receive threshold (RXThresh) & $-85 \mathrm{dBm}$ \\
\hline Carrier-sense threshold (CSThresh) & $-92 \mathrm{GHz}$ \\
\hline System loss (L) & 1 \\
\hline Antenna type & Omnidirectional \\
\hline Antenna gain (Gt, Gr) & 1 \\
\hline Antenna height (Z) & $1.5 \mathrm{~m}$ \\
\hline \multirow{2}{*}{ Propagation model: shadowing } & path loss exponent $=2.5$ \\
\cline { 2 - 2 } & shadowing deviation $=4.0 \mathrm{~dB}$ \\
\hline Data traffic & Constant-bit rate (CBR) \\
\hline Data frame size & 30 bytes \\
\hline Data traffic period & from 5 seconds to 60 seconds \\
\hline Maximum send queue size & 20 frames \\
\hline Number of topology & 10 \\
\hline Number of repetitions per topology & 10 \\
\hline Simulation duration & 3600 seconds \\
\hline
\end{tabular}

\subsection{Simulation results}
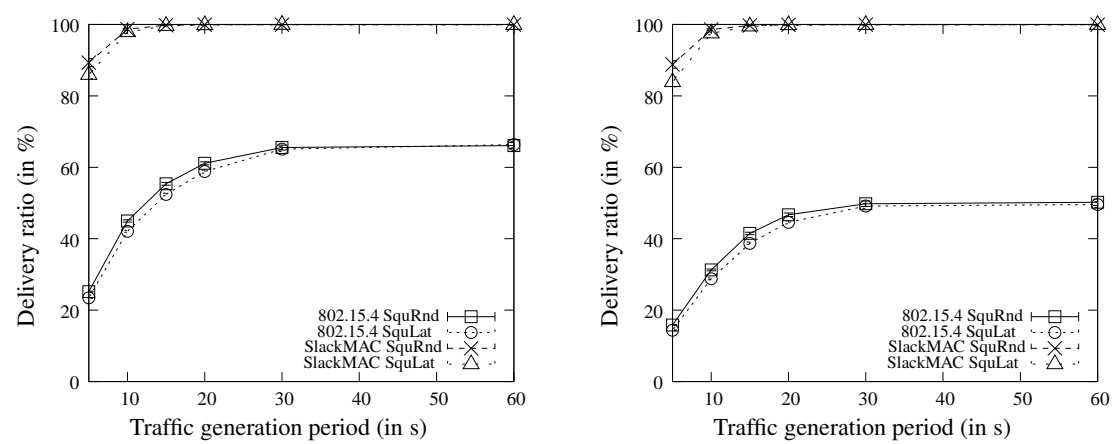

Fig. 4: The packets delivery ratio as a function of the traffic generation period with respectively the capture threshold of $2 \mathrm{~dB}$ (left) and $10 \mathrm{~dB}$ (right) for IEEE 802.15.4 and SlackMAC when square topologies are into grid and random.

Results with square topologies of size $250 \mathrm{~m} \times 250 \mathrm{~m}$ In this first scenario, we perform tests using both a grid and random topologies (respectively noted SquLat and SquRnd). We vary the traffic generation period from 5 seconds to 60 seconds with capture threshold of $2 \mathrm{~dB}$ and $10 \mathrm{~dB}$. 
Figures 4 and 5 show respectively the delivery ratio and the end-to-end delay of data frames, as a function of the traffic generation period (from $5 \mathrm{~s}$ to $60 \mathrm{~s}$ ) for IEEE 802.15.4 and SlackMAC, when square topologies are into grid and random.
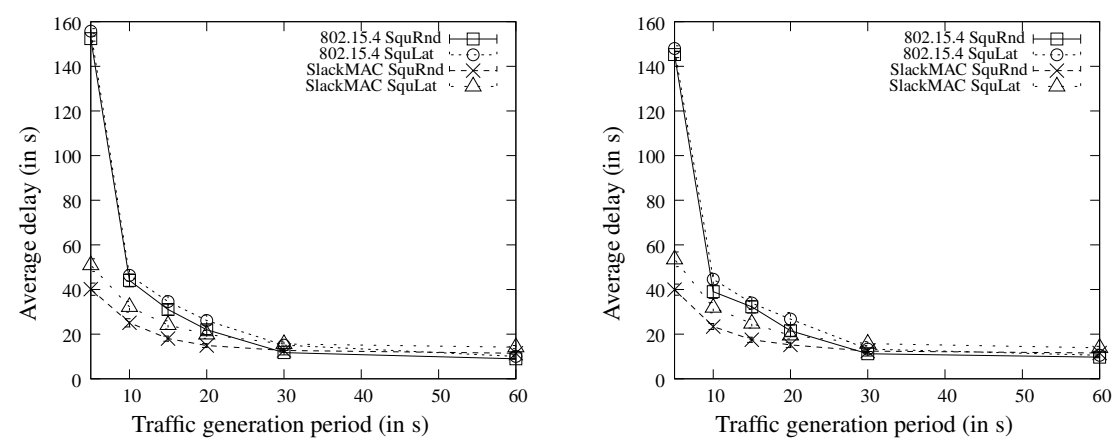

Fig. 5: Average delay as a function of the traffic generation period with respectively the capture threshold of $2 \mathrm{~dB}$ (left) and $10 \mathrm{~dB}$ (right) for IEEE 802.15.4 and SlackMAC when square topologies are into grid and random.

\section{For IEEE 802.15.4:}

- when nodes are positioned in a grid, the delivery ratio increases from $23.43 \%$ to $66.34 \%$ for the capture threshold of $2 \mathrm{~dB}$ and from $14.37 \%$ to $49.56 \%$ for the capture threshold of $10 \mathrm{~dB}$. The average delay decreases from $155.75 \mathrm{~s}$ to $10.02 \mathrm{~s}$ for the capture threshold of $2 \mathrm{~dB}$ and from $148 \mathrm{~s}$ to $10.52 \mathrm{~s}$ for the capture threshold of $10 \mathrm{~dB}$.

- when nodes are randomly positioned, the delivery ratio increases from $25.18 \%$ to $66.07 \%$ for the capture threshold of $2 \mathrm{~dB}$ and from $15.81 \%$ to $50.2 \%$ for the capture threshold of $10 \mathrm{~dB}$. The average delay decreases from $144.69 \mathrm{~s}$ to $7.22 \mathrm{~s}$ for the capture threshold of $2 \mathrm{~dB}$ and from $145.28 \mathrm{~s}$ to $9.66 \mathrm{~s}$ for the capture threshold of $10 \mathrm{~dB}$.

For SlackMAC:

- when nodes are positioned in a grid, the delivery ratio increases from $85.91 \%$ to $99.07 \%$ for the capture threshold of $2 \mathrm{~dB}$ and from $83.89 \%$ to $99.9 \%$ for the capture threshold of $10 \mathrm{~dB}$. The average delay decreases from $59.09 \mathrm{~s}$ to $14.19 \mathrm{~s}$ for the capture threshold of $2 \mathrm{~dB}$ and from $53.52 \mathrm{~s}$ to $13.90 \mathrm{~s}$ for the capture threshold of $10 \mathrm{~dB}$.

- when nodes are randomly positioned, the delivery ratio increases from $89.29 \%$ to $99.93 \%$ for the capture threshold of $2 \mathrm{~dB}$ and from $88.08 \%$ to $99.92 \%$ for the capture threshold of $10 \mathrm{~dB}$. The average delay decreases from $40.14 \mathrm{~s}$ to $11.47 \mathrm{~s}$ for the capture threshold of $2 \mathrm{~dB}$ and from $39.96 \mathrm{~s}$ to $11.55 \mathrm{~s}$ for the capture threshold of $10 \mathrm{~dB}$.

The results of this scenario show that for SlackMAC, whether the nodes are positioned in grid or randomly on a square area, the delivery ratio vary very little according to the traffic and the capture threshold. However, the average delay is better in the case of random positioning when the traffic varies regardless of the capture threshold. For the standard, the results also vary very little with the type of positioning. On the other hand, there is a difference of $16 \%$ in the delivery ratio between a capture threshold of 2 
$\mathrm{dB}$ and $10 \mathrm{~dB}$ for the two types of positioning. The average delay also varies little, but remains better when the positioning is random.

Results with pseudo-linear topologies of size $625 \mathrm{~m} \times 100 \mathrm{~m}$ In this second scenario, we perform tests using both a grid and random topologies (respectively noted StrLat and StrRnd). We also vary the traffic generation period from 5 seconds to 60 seconds with capture threshold of $2 \mathrm{~dB}$ and $10 \mathrm{~dB}$.
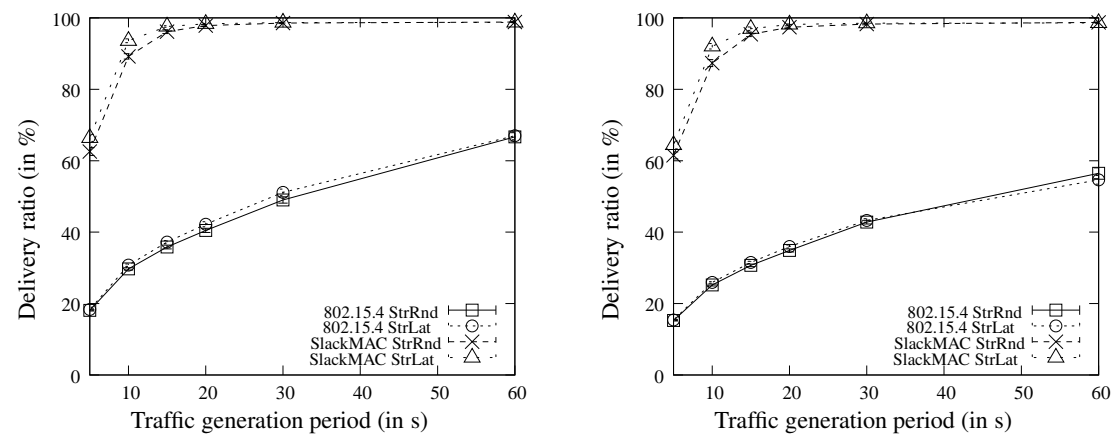

Fig. 6: The packets delivery ratio as a function of the traffic generation period with respectively the capture threshold of $2 \mathrm{~dB}$ (left) and $10 \mathrm{~dB}$ (right) for IEEE 802.15.4 and SlackMAC when pseudo-linear topologies are into grid and random.

Figures 6 and 7 show respectively the delivery ratio and the end-to-end delay of data frames, as a function of the traffic generation period (from $5 \mathrm{~s}$ to $60 \mathrm{~s}$ ) for IEEE 802.15.4 and SlackMAC, when pseudo-linear topologies are into grid and random.
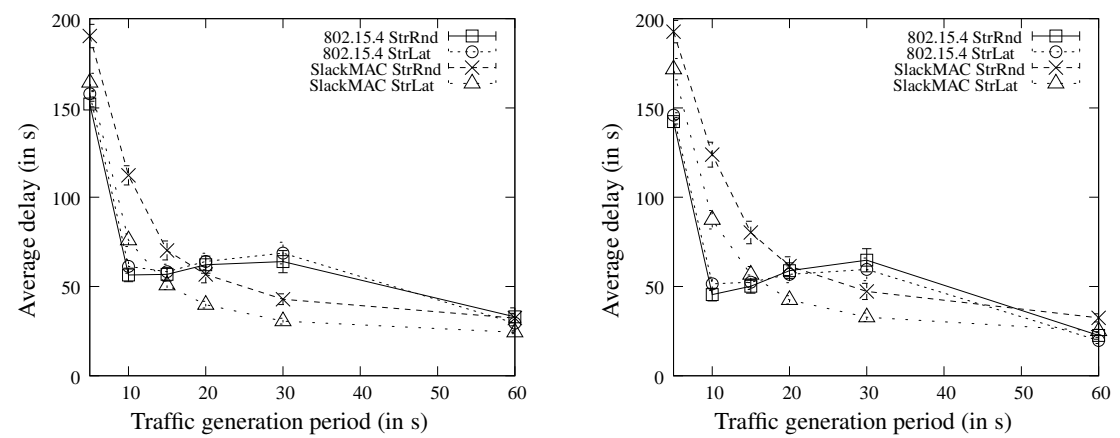

Fig. 7: Average delay as a function of the traffic generation period with respectively the capture threshold of $2 \mathrm{~dB}$ (left) and $10 \mathrm{~dB}$ (right) for IEEE 802.15.4 and SlackMAC when pseudo-linear topologies are into grid and random. 
For IEEE 802.15.4:

- when nodes are positioned in a grid, the delivery ratio increases from $18.34 \%$ to $66.98 \%$ for the capture threshold of $2 \mathrm{~dB}$ and from $15.42 \%$ to $54.65 \%$ for the capture threshold of $10 \mathrm{~dB}$. The average delay decreases from $157.94 \mathrm{~s}$ to $29.49 \mathrm{~s}$ for the capture threshold of $2 \mathrm{~dB}$ and from $145.99 \mathrm{~s}$ to $19.79 \mathrm{~s}$ for the capture threshold of $10 \mathrm{~dB}$.

- when nodes are randomly positioned, the delivery ratio increases from $18.09 \%$ to $66.67 \%$ for the capture threshold of $2 \mathrm{~dB}$ and from $15.28 \%$ to $56.48 \%$ for the capture threshold of $10 \mathrm{~dB}$. The average delay decreases from $152.20 \mathrm{~s}$ to $33.02 \mathrm{~s}$ for the capture threshold of $2 \mathrm{~dB}$ and from $142.32 \mathrm{~s}$ to $22.59 \mathrm{~s}$ for the capture threshold of $10 \mathrm{~dB}$.

For SlackMAC:

- when nodes are positioned in a grid, the delivery ratio increases from $66.40 \%$ to $98.72 \%$ for the capture threshold of $2 \mathrm{~dB}$ and from $64.41 \%$ to $98.57 \%$ for the capture threshold of $10 \mathrm{~dB}$. The average delay decreases from $164.26 \mathrm{~s}$ to $24.39 \mathrm{~s}$ for the capture threshold of $2 \mathrm{~dB}$ and from $171.67 \mathrm{~s}$ to $25.10 \mathrm{~s}$ for the capture threshold of $10 \mathrm{~dB}$.

- when nodes are randomly positioned, the delivery ratio increases from $62.55 \%$ to $98.8 \%$ for the capture threshold of $2 \mathrm{~dB}$ and from $61.49 \%$ to $98.73 \%$ for the capture threshold of $10 \mathrm{~dB}$. The average delay decreases from $190.27 \mathrm{~s}$ to $32.32 \mathrm{~s}$ for the capture threshold of $2 \mathrm{~dB}$ and from $192.74 \mathrm{~s}$ to $32.43 \mathrm{~s}$ for the capture threshold of $10 \mathrm{~dB}$.

The results of this second scenario show that for SlackMAC, whether the nodes are positioned in grid or randomly on a square area, the delivery ratio vary very little according to the traffic and the capture threshold. However, unlike the square zone where the best average delay is obtained with a random positioning, that is not the case with a pseudo-linear zone in which the best average delay is with a grid positioning regardless of the capture threshold. For the standard, the results vary very little with the type of positioning for the delivery ratio and the average delay regardless of the capture threshold.

Effect of capture threshold In this last scenario, we perform tests using the two topologies area such as square and pseudo-linear. We set the traffic generation period to 60 seconds and vary the capture threshold from $2 \mathrm{~dB}$ to $10 \mathrm{~dB}$.

Figures 8 and 9 show respectively the delivery ratio and the end-to-end delay of data frames, as a function of the capture threshold (from $2 \mathrm{~dB}$ to $10 \mathrm{~dB}$ ) for IEEE 802.15.4 and SlackMAC.

For IEEE 802.15.4:

- when topology is square, the delivery ratio decreases from $66.34 \%$ to $49.56 \%$ for grid positioning and from $66.07 \%$ to $50.20 \%$ for random positioning. The average delay increases from $10.02 \mathrm{~s}$ to $10.52 \mathrm{~s}$ for grid positioning and from $8.93 \mathrm{~s}$ to $9.66 \mathrm{~s}$ for random positioning.

- when topology is pseudo-linear, the delivery ratio decreases from $66.98 \%$ to $54.65 \%$ for grid positioning and from $66.67 \%$ to $56.48 \%$ for random positioning. The average delay decreases from $29.49 \mathrm{~s}$ to $19.79 \mathrm{~s}$ for grid positioning and from $33.02 \mathrm{~s}$ to $22.59 \mathrm{~s}$ for random positioning.

For SlackMAC: 

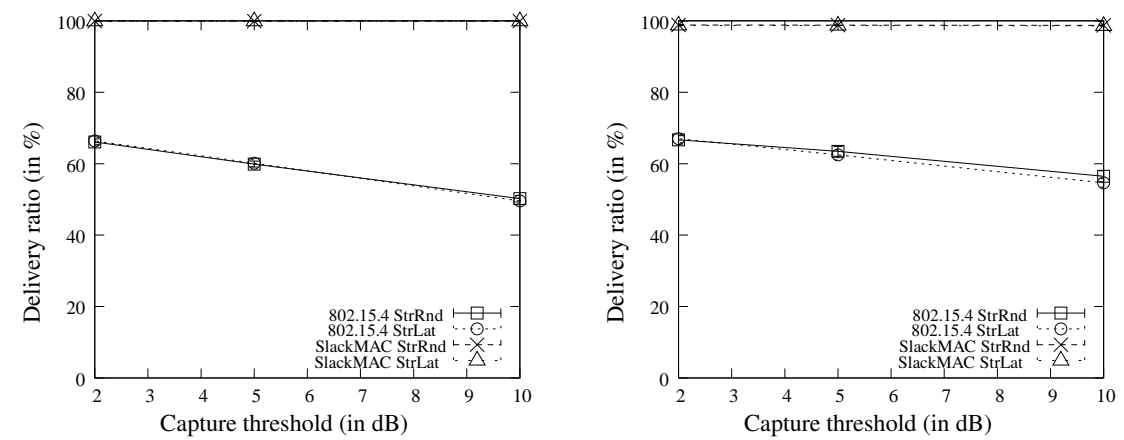

Fig. 8: The packets delivery ratio as a function of the capture threshold with respectively square topology (left) and pseudo-linear topology (right) into grid and random for IEEE 802.15.4 and SlackMAC when the traffic generation period is 60 seconds.
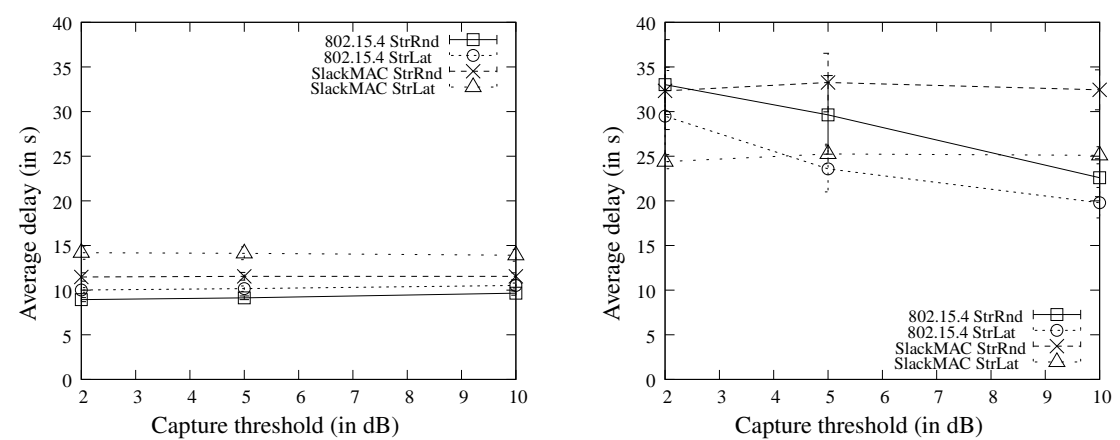

Fig. 9: Average delay as a function of the capture threshold with respectively square topology (left) and pseudo-linear topology (right) into grid and random for IEEE 802.15.4 and SlackMAC when the traffic generation period is 60 seconds. 
- when topology is square, the delivery ratio is always around $99.9 \%$ for grid positioning and for random positioning. The average delay decreases slowly from $14.19 \mathrm{~s}$ to $13.9 \mathrm{~s}$ for grid positioning and always around $11 \mathrm{~s}$ for random positioning.

- when topology is pseudo-linear, the delivery ratio is always around $98.8 \%$ for grid positioning and for random positioning. The average delay decreases slowly from $24.39 \mathrm{~s}$ to $25.10 \mathrm{~s}$ for grid positioning and always around $32 \mathrm{~s}$ for random positioning.

The results of this last scenario show that for SlackMAC, whether the nodes are positioned in grid or randomly for the two zones of topology the delivery ratio varies little with the capture threshold. However, the average delay for a square zone is better for random positioning than grid positioning and for a pseudo-linear zone the better average delay is obtained with a grid positioning. Unlike SlackMAC, the delivery ratio in the standard decreases when the capture threshold increases regardless of the zone and the positioning. The average delay increases with the capture threshold for square zone and decreases with the capture threshold for pseudo-linear zone regardless of positioning.

\section{Conclusion}

In this work, we show the need to be attentive and precise about the description of the simulation conditions comparing the performance of communication protocols for WSNs. For a given and widespread signal propagation model we pointed out how the topology choice and the capture threshold value may impact simulations results. To do this we used an asynchronous MAC protocol SlackMAC and the reference MAC protocol specified in the standard IEEE 802.15.4 in beacon-enabled mode. The aim of the paper is not to make an exhaustive comparison of protocols. We knew intuitively that the asynchronous MAC protocol would provide better overall performance than the standard. What we have demonstrated with intensive simulations, is the diversity in the results when the simulation conditions change. The conditions we have dealt with are the topology types and the capture threshold used in a WSN simulation process : (i) grid or random topologies for squared or stretched areas and (ii) capture threshold varying from $2 \mathrm{~dB}$ to $10 \mathrm{~dB}$. The results showed great diversity such as for example for the same form of topology (square or pseudo-linear), either one of the two protocols gives better performances when the positioning is in grid and the other one rather with a random positioning. These observations are also made for two different forms of topology. These results also showed a very significant impact of the capture threshold on one protocol than the other. These remarkable differences between performances when simulation conditions change, confirm that it is essential to clearly define the simulation environment and also to reconcile the chosen conditions with the results when comparing the performances of protocols.

\section{References}

1. IEEE 802.15, "IEEE standard for local and metropolitan area networks - part 15.4: Lowrate wireless personal area networks (LR-WPANs), ' ANSI/IEEE, Standard 802.15.4 R2011, 2011. 
2. A. M. Kanthe, D. Simunic, and R. Prasad, "Effects of propagation models on AODV in mobile ad-hoc networks," Wireless personal communications, vol. 79, no. 1, pp. 389-403, 2014.

3. M. Alduais and N. Abdulwahab, "The performance evaluation of different logical topologies and their respective protocols for wireless sensor networks," Ph.D. dissertation, Universiti Tun Hussein Onn Malaysia, 2015.

4. A. T. Aby, A. Guitton, P. Lafourcade, and M. Misson, "History-based MAC protocol for low duty-cycle wireless sensor networks: the Slack-MAC protocol," in EAI Endorsed Transactions on Mobile Communications and Applications. EUDL: European Union Digital Library, 2016.

5. - "Slack-MAC: Adaptive MAC protocol for low duty-cycle wireless sensor networks," in Ad Hoc Networks. Springer, 2015, pp. 69-81.

6. G. Chalhoub, A. Guitton, and M. Misson, "MAC specifications for a WPAN allowing both energy saving and guaranted delay - Part A: MaCARI: a synchronized tree-based MAC protocol," in IFIP WSAN, 2008.

7. K. Nguyen, Y. Ji, and S. Yamada, "Low overhead MAC protocol for low data rate wireless sensor networks," International Journal of Distributed Sensor Networks, 2013.

8. W. Ye, J. Heidemann, and D. Estrin, "An energy-efficient mac protocol for wireless sensor networks," in INFOCOM 2002. Twenty-First Annual Joint Conference of the IEEE Computer and Communications Societies. Proceedings. IEEE, vol. 3. IEEE, 2002, pp. 1567-1576.

9. G. Lu, B. Krishnamachari, and C. Raghavendra, "An adaptive energy-efficient and lowlatency MAC for tree-based data gathering in sensor networks," in Wireless Communications and Mobile Computing, vol. 7, September 2007, pp. 863-875.

10. W. Z. Song, R. Huang, B. Shirazi, and R. LaHusen, "TreeMAC: Localized TDMA protocol for real-time high-data-rate sensor networks," vol. 5, no. 6, 2009, pp. 750-765.

11. F. D. Cunha, I. Cunha, H. C. Wong, A. A. Loureiro, and L. B. Oliveira, "ID-MAC: An identity-based MAC protocol for wireless sensor networks," in Computers and Communications (ISCC), 2013 IEEE Symposium on. IEEE, 2013, pp. 000 975-000 981.

12. Y. Sun, S. Du, O. Gurewitz, and D. B. Johnson, "DW-MAC: a low latency, energy efficient demand-wakeup MAC protocol for wireless sensor networks," in Proceedings of the 9th ACM international symposium on Mobile ad hoc networking and computing. ACM, 2008, pp. 53-62.

13. Y. Gu and T. He, "Dynamic switching-based data forwarding for low-duty-cycle wireless sensor networks," IEEE Transactions on Mobile Computing, vol. 10, no. 12, pp. 1741-1754, 2011.

14. L. Cheng, Y. Gu, J. Niu, T. Zhu, C. Liu, Q. Zhang, and T. Hel, "Taming collisions for delay reduction in low-duty-cycle wireless sensor networks," in IEEE INFOCOM 2016-The 35th Annual IEEE International Conference on. IEEE, 2016, pp. 1-9.

15. J. Polastre, J. Hill, and D. Culler, "Versatile low power media access for wireless sensor networks," in ACM Sensys, November 2004.

16. M. Buettner, Y. Gary, V., E. Anderson, and R. Han, "X-MAC: A short preamble MAC protocol for duty-cycled wireless sensor networks," in Proceedings of the 4th international conference on Embedded networked sensor systems. ACM, 2006, pp. 307-320.

17. D. Moss and P. Levis, "BoX-MACs: Exploiting physical and link layer boundaries in lowpower networking," Computer Systems Laboratory Stanford University, pp. 116-119, 2008.

18. G. Kim and J. Ahn, "On-demand synchronous X-MAC protocol," in Computer Science and Software Engineering (JCSSE). IEEE, 2016, pp. 1-6.

19. Y. Sun, O. Gurewitz, and D. B. Johnson, "RI-MAC: a receiver-initiated asynchronous duty cycle MAC protocol for dynamic traffic loads in wireless sensor networks," in ACM Sensys, 2008. 
20. I. Park, H. Lee, and S. Kang, "RIX-MAC: An energy-efficient receiver-initiated wakeup MAC protocol for WSNs." KSII Transactions on Internet \& Information Systems, vol. 8, no. 5, 2014.

21. K. Nguyen, V. Nguyen, D. Le, Y. Ji, D. A. Duong, and S. Yamada, “A receiver-initiated MAC protocol for energy harvesting sensor networks," in Ubiquitous Information Technologies and Applications. Springer, 2014, pp. 603-610.

22. X. Wang, X. Zhang, G. Chen, and Q. Zhang, "Opportunistic cooperation in low duty cycle wireless sensor networks," in Communications (ICC), 2010 IEEE International Conference on. IEEE, 2010, pp. 1-5.

23. H. Yoo, M. Shim, and D. Kim, "Dynamic duty-cycle scheduling schemes for energyharvesting wireless sensor networks," IEEE communications letters, vol. 16, no. 2, pp. 202 204, 2012.

24. A. T. Aby, A. Guitton, and M. Misson, "Study of blind rendez-vous in low power wireless sensor networks," in Vehicular Technology Conference (VTC Spring), 2014 IEEE 79th. IEEE, 2014, pp. 1-5.

25. "Network simulator 2," 2002, http://www.isi.edu/nsnam/ns.

26. M. S. Mezghanni, N. Kandil, and N. Hakem, "Performance study of ieee 802.15. 4/4g waveforms over the mobile underground mine radio-channel," in Vehicular Technology Conference (VTC-Fall), IEEE 84th. IEEE, 2016, pp. 1-6.

27. M. K. Simon and M. Alouini, "Outage performance of multiuser communication systems," Digital Communication over Fading Channels, Second Edition, pp. 638-680, 2005. 